\title{
A simple and quantum-mechanically motivated characterization of the formally real Jordan algebras
}

\author{
Gerd Niestegge \\ gerd.niestegge@web.de
}

\begin{abstract}
Quantum theory's Hilbert space apparatus in its finite-dimensional version is nearly reconstructed from four simple and quantum-mechanically motivated postulates for a quantum logic. The reconstruction process is not complete, since it excludes the two-dimensional Hilbert space and still includes the exceptional Jordan algebras, which are not part of the Hilbert space apparatus. Options for physically meaningful potential generalizations of the apparatus are discussed.
\end{abstract}

Key Words: Jordan algebra; quantum logic; transition probability; higher-order interference

PACS: 03.65.Ta, 02.30.Tb

MSC: $17 \mathrm{C} 55 ; 81 \mathrm{P} 10 ; 81 \mathrm{P} 16$

\section{Introduction}

Quantum mechanics needs the vast mathematical Hilbert space formalism, but does not provide a plausible derivation for it. Attempts to derive this formalism, including its statistical interpretation, from a small number of simple and plausible postulates have a long history. The focus changed from the algebraic and quantum logical approaches in earlier years [10, 21, 24, 30, 34, 49, 53, 55, 57, 62, 63 to the operational and information theoretic approaches, often using the generalized probabilistic theories or sometimes category theory as their framework, in later years [5, 6, 7, 13, 14, 16, 17, 20, 26, 29, 32, 33, 35, 54, 60, 61, 64.

The present paper resumes the quantum logical approach, considering an abstract model of projective quantum measurement therein. A major postulate becomes the non-existence of third-order interference. Third-order interference and its absence in quantum mechanics were discovered by Sorkin in 1994 [59] and were not known in the earlier years. This has become a matter of experimental and theoretical research in the recent past [6, 7, 15, 28, 31, 45, 48, 56, 58. 
Selecting two further basic features of quantum mechanics as postulates, it is shown that, in the finite-dimensional case, the quantum logic can be represented as the system of idempotent elements in a formally real Jordan algebra; this type of algebra was introduced and classified by J. von Neumann, P. Jordan and E. Wigner 63.

This paper reuses some of the author's earlier results [44. These results also apply in the infinite-dimensional case, but the restriction to finite dimensions in the present paper renders possible to sharpen the result (in [44, the quantum logic is only dense in, but not identical to the system of idempotent elements) as well as to significantly simplify the postulates and to introduce them immediately into the framework of the quantum logic and its states. Some postulates needed in 44 (power-associativity and positivity of squares) require the derivation of some additional structure (an algebra which is neither commutative nor associative in the general case and which will be used later to prove the major results in section 5) before they can be defined; in the present paper, these postulates are derived from different and simpler ones.

Most recent operational and information theoretic approaches are also restricted to the finite-dimensional case. Moreover, they often include only the simple (or irreducible) Jordan algebras, although some quantum-mechanical applications (e.g., superselection) require the use of non-simple algebras of observables. The approach of the present paper includes the non-simple case. Furthermore, it differs from all other papers in the usage of one special postulate: the existence and the uniqueness of the conditional probabilities on the quantum logic (which turn out to become the abstract model of projective quantum measurement mentioned above).

The postulates are introduced in the next section. After a brief outline of the formally real Jordan algebras in section 3, some auxiliary results are derived from the postulates in section 4 . The main results are presented and proven in section 5. Options for physically reasonable potential generalizations of the mathematical apparatus of quantum theory are discussed in the concluding section.

\section{The four postulates}

The quantum logic of the usual quantum mechanics consists of the observables with the simple spectrum $\{0,1\}$ (or, equivalently, of the self-adjoint projections or of the closed linear subspaces of the Hilbert space) and forms an orthomodular lattice. Originally, therefore, a quantum logic was mostly assumed to be an orthomodular lattice. However, there is no physical motivation for the existence of lattice operations for propositions which are not compatible, and later a quantum logic was often assumed to be an orthomodular partially ordered set only. So this is what is done here. Different more general structures are conceivable and one was sometimes used in the author's previous work; however, they involve more mathematical subtleties, which shall be avoided here. 
In this paper, a quantum logic shall be an orthomodular partially ordered set $L$ with order relation $\leq$, smallest element 0 , largest element $\mathbb{I}$ and an orthocomplementation'. This means that the following conditions are satisfied by the $p, q \in L$ :

(a) $q \leq p$ implies $p^{\prime} \leq q^{\prime}$.

(b) $\left(p^{\prime}\right)^{\prime}=p$.

(c) $p \leq q^{\prime}$ implies that $p \vee q$, the supremum of $p$ and $q$, exists.

(d) $p \vee p^{\prime}=\mathbb{I}$.

(e) Orthomodular law: $q \leq p$ implies $p=q \vee\left(p \wedge q^{\prime}\right)$.

Here, $p \wedge q$ denotes the infimum of $p$ and $q$, which exists iff $p^{\prime} \vee p^{\prime}$ exists.

The elements of the quantum logic are called propositions. A proposition $e$ is called minimal if there is no proposition $q$ with $q \leq e$ and $0 \neq q \neq e$. The minimal propositions are also called atoms in the common literature. Two propositions $p$ and $q$ are orthogonal, if $p \leq q^{\prime}$ or, equivalently, $q \leq p^{\prime}$; in this case, $p \vee q$ shall be noted by $p+q$ in the following. The interpretation of this mathematical terminology is as follows: orthogonal events are exclusive, $p^{\prime}$ is the negation of $p$, and $p+q:=p \vee q$ is the disjunction of the two exclusive events, $p$ and $q$.

It is not assumed either that the quantum logic $L$ is a lattice (in a lattice, $p \vee q$ and $p \wedge q$ exist for all lattice elements $p$ and $q$ ) or that it satisfies the so-called covering property. Both play important roles in the early quantum logical approaches [10, 30, 49, 57, 62.

Let $V$ denote the linear space of the bounded real-valued functions on $L$, which are additive for orthogonal propositions. A state allocates probability values to the propositions and is an element $\mu \in V$ with $\mu(p) \geq 0$ for all $p \in L$ and $\mu(\mathbb{I})=1$.

Suppose a state $\mu$ and a proposition $p$ with $\mu(p) \neq 0$. If one wishes to extend the usual conditional probabilities from standard probability theory to this setting, the minimum requirement for the conditioned state $\mu_{p}$ of $\mu$ under $p$ is that

$$
\mu_{p}(q)=\frac{1}{\mu(p)} \mu(q) \text { for } q \leq p .
$$

In the following, it shall be assumed that such a state $\mu_{p}$ exists for each state $\mu$ and each proposition $p$ with $\mu(p) \neq 0$ and that it is unique (i.e. there is only one such state $\mu_{p}$ ).

Definition 2.1. In accordance with the usual case, the conditional probability of a proposition $q$ under a further proposition $p$ in a state $\mu$ with $\mu(p) \neq 0$ is denoted by

$$
\mu(q \mid p):=\mu_{p}(q) .
$$

The assumption that unique conditional probabilities exist does not hold for many quantum logics, but it does hold for the Hilbert space quantum logic 
used in quantum mechanics (except for the two-dimensional case), and it turns out that the above transition from $\mu$ to the conditioned state then becomes identical to the state transition of the projective quantum measurement (Lüders - von Neumann measurement; see 42, 43. and also remark 3.2 in section 3). On the one hand, this provides a strong quantum-mechanical motivation for the above extension of conditional probability. On the other hand, it is quite surprising that a natural extension of the classical conditional probability from the Boolean lattices to the quantum logics considered here brings us to quantum measurement, the behavior of which is far away from any classical conceptions and the source of many well-known interpretational and philosophical troubles with quantum mechanics.

That projective quantum measurement is the appropriate rule for conditionalizing probabilities in the non-Boolean quantum logic of quantum mechanics has already been pointed out by J. Bub 11] and others 18. Conditional probabilities, although not always defined in the same way as above, were considered in the early attempts to derive the quantum-mechanical formalism [22, 24, 50, but their existence and uniqueness was used as a postulate for the first time in 2001 in 42. Further various ways of introducing conditional probabilities on quantum logics can be found in [38, 39, 40, 41, 51, 52.

In the case of a minimal proposition $e$, the state $\mu_{e}: q \rightarrow \mu(q \mid e), q \in L$, becomes independent of $\mu$ and is the unique state allocating the probability 1 to the minimal proposition $e$.

Definition 2.2. Let $e \in L$ be a minimal proposition. The state-independent conditional probability of the propositions $q \in L$ under $e$ is denoted by $\mathbb{P}(q \mid e)$; this means that the following identity holds for all states $\mu$ with $\mu(e) \neq 0$ and all propositions $q \in L$ :

$$
\mathbb{P}(q \mid e)=\mu(q \mid e)
$$

The values of $\mathbb{P}(\mid)$ are intrinsic properties of the algebraic structure of the quantum logic and not primarily of its state space; they are invariant under the algebraic automorphisms of the quantum logic. Furthermore, $\mathbb{P}(q \mid e)=1$ for any $q \in L$ with $e \leq q$, and $\mathbb{P}(q \mid e)=0$ for any $q \in L$ with $e \leq q^{\prime}$ (i.e., $e$ and $q$ are orthogonal). Indeed, 0 and 1 are the only values which $\mathbb{P}(q \mid e)$ can assume in a classical (i.e., Boolean) logic, while any value in the unit interval is assumed in the quantum case.

The minimal propositions in the Hilbert space quantum logic are the lines in the Hilbert space, and the transition probability becomes $\mathbb{P}\left(e_{2} \mid e_{1}\right)=\cos ^{2} \theta$, where $\theta$ is the angle between the two lines $e_{1}$ and $e_{2}$ 42, 43. We then have $\mathbb{P}\left(e_{2} \mid e_{1}\right)=\mathbb{P}\left(e_{1} \mid e_{2}\right)$. This interesting symmetry featured by the quantummechanical transition probabilities will also become one of the postulates in this paper. A similar axiom was used in many other studies, such as [1, 3, 24].

Moreover, the lines in the Hilbert space represent the quantum-mechanical pure states, and the mixed states can be expressed as combinations of orthogonal pure states, which motivates the assumption that the elements in $V$ are combinations of state-independent conditional probabilities generated by some orthogonal minimal propositions. A similar assumption occurs in [5, 7]; they do 
not use the state-independent conditional probabilities, but pure states instead, and postulate the orthogonal decomposition only for the states and not for all elements of $V$.

A further feature of quantum mechanics is the impossibility of third-order interference, which was discovered by Sorkin [59]. Third-order interference is motivated by the consideration of three-slit experiments and the question of whether they involve a new type of interference versus the common two-slit experiments. This can be expressed with the conditional probabilities in the following way.

$$
\begin{aligned}
& \mu\left(q \mid p_{1}+p_{2}+p_{3}\right) \mu\left(p_{1}+p_{2}+p_{3}\right) \\
& -\mu\left(q \mid p_{1}+p_{2}\right) \mu\left(p_{1}+p_{2}\right)-\mu\left(q \mid p_{1}+p_{3}\right) \mu\left(p_{1}+p_{3}\right)-\mu\left(q \mid p_{2}+p_{3}\right) \mu\left(p_{2}+p_{3}\right) \\
& +\mu\left(q \mid p_{1}\right) \mu\left(p_{1}\right)+\mu\left(q \mid p_{2}\right) \mu\left(p_{2}\right)+\mu\left(q \mid p_{3}\right) \mu\left(p_{3}\right),
\end{aligned}
$$

where $p_{1}, p_{2}, p_{3}$ are three orthogonal propositions, $q$ is a further proposition and $\mu$ is a state. Third-order interference means that there are states and propositions making this term non-zero.

The four postulates that the quantum logic $L$ shall satisfy can now be stated in the following way.

(A) For every pair of propositions $p$ and $q$ in $L$ with $p \neq q$, there is a state $\mu$ with $\mu(p) \neq \mu(q)$. The (extended) conditional probabilities exist and are unique.

(B) The dimension of $V$ is finite, and each $\mu \in V$ can be written as

$$
\mu(p)=\sum_{k=1}^{m} r_{k} \mathbb{P}\left(p \mid e_{k}\right), p \in L,
$$

with some orthogonal minimal propositions $e_{1}, \ldots, e_{m}$, real numbers $r_{1}, \ldots, r_{m}$ and some positive integer $m$.

(C) For any two minimal propositions $e$ and $f$ in $L$, the transition probability satisfies the symmetry condition

$$
\mathbb{P}(f \mid e)=\mathbb{P}(e \mid f)
$$

(D) Third-order interference does not occur.

In [7, the non-existence of third-order interference is combined with a certain strong symmetry postulate, which implies the symmetry for the transition probabilities (C) considered here and which rules out the non-simple or reducible Jordan algebras (except for the classical case). In [5], it is shown that the nonexistence of third-order interference is a redundant postulate in [7. Regardless of the different frameworks, the present paper takes another approach: keeping the non-existence of third-order interference and using the weaker symmetry postulate (C), Jordan algebras can be derived in such a way that the non-simple or reducible ones are included. 


\section{Jordan algebras}

A Jordan algebra is a linear space $A$ with a commutative bilinear product 0 satisfying the identity $\left(x^{2} \circ y\right) \circ x=x^{2} \circ(y \circ x)$ for $x, y \in A$. A Jordan algebra over the real numbers is called formally real, if $x_{1}^{2}+\ldots+x_{m}^{2}=0$ implies $x_{1}=\ldots=x_{m}=0$ for any $x_{1}, \ldots, x_{m} \in A$ and any positive integer $m$. In the finite-dimensional case, the formally real Jordan algebras coincide with the so-called JB-algebras and JBW-algebras [1, 25].

Each finite-dimensional formally real Jordan algebra possesses a multiplicative identity $\mathbb{I}$ and a natural order relation; the system of its idempotent elements becomes a quantum logic with $p^{\prime}:=\mathbb{I}-p$.

A finite-dimensional formally real Jordan algebra decomposes into a direct sum of simple or irreducible subalgebras, which are either one-dimensional (the real numbers), spin factors (a spin factor is characterized by the conditions that all propositions in the quantum logic formed by its idempotent elements are either minimal or identical to 0 or $\mathbb{I}$ and that there are at least three different minimal propositions) or can be represented as algebras of the Hermitian $k \times k$-matrices over the real numbers, complex numbers, quaternions with $k=3,4,5, \ldots$ or over the octonions with $k=3$ only [25, 63]. The product for the matrices $x, y$ is given by $x \circ y:=(x y+y x) / 2$.

Almost all finite-dimensional formally real Jordan algebras have a representation as a Jordan subalgebra of a complex matrix algebra or, equivalently, the self-adjoint operators on a unitary space. Such a representation is not possible only for the so-called exceptional algebras; these are particularly the algebra that consists of Hermitian $3 \times 3$-matrices over the octonions and all algebras that include this one as one of their irreducible subalgebras [25.

Proposition 3.1. The system of idempotent elements in a finite-dimensional formally real Jordan algebra A yields a quantum logic satisfying the above postulates (A), (B), (C) and (D), if none of the irreducible subalgebras of $A$ is a spin factor.

Proof. Let $A$ be a finite-dimensional formally real Jordan algebra such that none of its irreducible subalgebras is a spin factor. In [42, it is shown that the unique conditional probabilities exist (postulate $(\mathrm{A})$ ) and have the shape

$$
\mu(q \mid p)=\frac{1}{\mu(p)} \mu(\{p, q, p\}),
$$

with idempotent elements $p$ and $q$ in $A$, a state $\mu$ and $\mu(p)>0$. Here, $\{,$, denotes the so-called Jordan triple product $\{x, y, z\}:=x \circ(y \circ z)-y \circ(z \circ x)+$ $z \circ(x \circ y)$ for $x, y, z \in A$. Moreover, the unique extension of the state $\mu$ from the idempotent elements to the whole algebra $A$, which exists by an extension of Gleason's theorem to Jordan algebras [12, 19, is used on the right-hand side of the above equation. 
The above special shape of the conditional probabilities implies that thirdorder interference does not exist (postulate (D)) and, moreover, that $\mathbb{P}(f \mid e)=r$ iff $\{e, f, e\}=r e$ for minimal propositions $e, f$ and $r \in \mathbb{R}$.

$A$ possesses a natural real-valued trace, defined by linearity and the condition that $\operatorname{trace}(e)=1$ holds for each minimal proposition $e$ [1]. It is identical to the trace inherited from the representation of $A$ as a direct sum of matrix algebras. It satisfies the identity $\operatorname{trace}(\{p, q, p\})=\operatorname{trace}(p \circ q)$ for idempotent elements $p$ and $q$ in $A$, which implies $\mathbb{P}(f \mid e)=\operatorname{trace}(e \circ f)=\operatorname{trace}(f \circ e)=\mathbb{P}(e \mid f)$ for minimal propositions $e$ and $f$; so we have $(\mathrm{C})$. Moreover, $A$ becomes a Hilbert space with the inner product $\langle x \mid y\rangle:=\operatorname{trace}(x \circ y), x, y \in A$. Hilbert spaces are self-dual and the spectral theorem for the formally real Jordan algebras then yields postulate (B).

Remark 3.2. To see the link to projective quantum measurement, which has already been mentioned above, note that the Jordan triple product $\{p, q, p\}$ coincides with the simple operator product $p q p$ in the case of the special Jordan product $x \circ y:=(x y+y x) / 2$ with Hilbert space operators $x$ and $y$. When the state $\mu$ is given by the statistical operator $a$ and $p, q$ are self-adjoint projection operators on a Hilbert space, equation (3.1) becomes

$$
\mu(q \mid p)=\frac{\mu(p q p)}{\mu(p)}=\frac{\operatorname{trace}(a p q p)}{\operatorname{trace}(a p)}=\frac{\operatorname{trace}(\text { papq })}{\operatorname{trace}(\text { pap })} .
$$

This shows that the state transition of projective quantum measurement (righthand side of (3.2)) is the same as the probability conditionalization (left-hand side of (3.2)) in the case of the Hilbert space quantum logic.

Proposition 3.3. If the irreducible subalgebras of a finite-dimensional formally real Jordan algebra A include a spin factor, the quantum logic formed by its idempotent elements does not possess unique conditional probabilities.

Proof. Since unique conditional probabilities on $A$ enforce unique conditional probabilities on each of its direct summands, it is sufficient to assume that $A$ itself is a spin factor. Let $L$ be the system of its idempotent elements, $e$ and $f$ minimal propositions that are neither identical nor orthogonal and $\mu$ a state with $\mu(e)=1$. Then define another state $\nu$ on $L$ that is identical to $\mu$ for all propositions except for $f$ and $f^{\prime}$. For these two propositions choose $\nu(f) \neq \mu(f)$ and $\nu\left(f^{\prime}\right)=1-\nu(f)$. Then $\nu(q)+\nu\left(q^{\prime}\right)=1$ for $q=0, q=\mathbb{I}$ and all minimal propositions $q$. Since $A$ is a spin factor, there are no further propositions and never more than two orthogonal non-zero propositions. Therefore $\nu$ indeed becomes a state on $L$ and so there are two different states $\mu \neq \nu$ with $\mu(e)=$ $1=\nu(e)$. This contradicts the uniqueness of the conditional probability, which implies that there is only one single state on $L$ allocating the probability 1 to a given minimal proposition (this is the state $p \rightarrow \mathbb{P}(p \mid e)$ ).

That a spin factor does not possess unique conditional probabilities is also an immediate consequence of the much more general lemma 3.3 in [42]. 


\section{Auxiliaries}

This section presents some simple auxiliary results, which will be used later and require only the first three postulates. The fourth one will be needed later.

Lemma 4.1. Let $L$ be a quantum logic satisfying the first three postulates (A), (B) and $(\mathrm{C})$.

(i) The cardinality of any family of pairwise orthogonal non-zero propositions in $L$ cannot exceed the dimension of $V$.

(ii) If $\sum_{i=1}^{l} e_{i} \leq \sum_{j=1}^{m} f_{j}$ with two families $e_{1}, \ldots, e_{l}$ and $f_{1}, \ldots, f_{m}$ of pairwise orthogonal minimal propositions, then $l \leq m$.

(iii) There is a minimal proposition $e$ in $L$ with $e \leq p$ for each proposition $p \neq 0$ in $L$.

(iv) Each proposition $p \neq 0$ in $L$ is the sum of a finite number of pairwise orthogonal minimal propositions.

(v) There is a unique state $\tau$ on $L$ with $\tau(e)=\tau(f)$ for all minimal propositions $e$ and $f$ in L. It is called the trace state and has the shape

$$
\tau(p)=\frac{1}{n} \sum_{k=1}^{n} \mathbb{P}\left(p \mid e_{k}\right),
$$

for $p \in L$, where $e_{1}, \ldots, e_{n}$ are any pairwise orthogonal minimal propositions with $\sum_{k=1}^{n} e_{k}=\mathbb{I}$.

(vi) The conditional probability in the trace state $\tau$ satisfies the identity

$$
\tau(q \mid p) \tau(p)+\tau\left(q \mid p^{\prime}\right) \tau\left(p^{\prime}\right)=\tau(q),
$$

for all propositions $q$ and $p \neq 0$ in $L$.

Proof. (i) Let $q_{j}$ be a family of pairwise orthogonal non-zero propositions in $L$. For each single $j$, there is a state allocating a non-zero value to $q_{j}$ due to postulate (A), and this state can be conditioned under $q_{j}$; thus we get a state $\mu_{j}$ with $\mu_{j}\left(q_{j}\right)=1$ for each $j$. Then $\mu_{j}\left(q_{i}\right)=0$ for $i \neq j$. Therefore, the states $\mu_{j}$ are linearly independent and the cardinality of both families $\mu_{j}$ and $q_{j}$ cannot exceed the dimension of $V$.

(ii) Let $e_{1}, \ldots, e_{l}$ and $f_{1}, \ldots, f_{m}$ be two families of pairwise orthogonal minimal propositions with $\sum_{i=1}^{l} e_{i} \leq \sum_{j=1}^{m} f_{j}$ and define a state $\mu$ by

$$
\mu(p):=\frac{1}{m} \sum_{j=1}^{m} \mathbb{P}\left(p \mid f_{j}\right) \text { for } p \in L .
$$

Then $e_{i} \leq \sum_{j=1}^{m} f_{j}$, hence $\mathbb{P}\left(\sum_{j=1}^{m} f_{j} \mid e_{i}\right)=1$ for each $i$ and by postulate $(\mathrm{C})$

$$
\mu\left(e_{i}\right)=\frac{1}{m} \sum_{j=1}^{m} \mathbb{P}\left(e_{i} \mid f_{j}\right)=\frac{1}{m} \sum_{j=1}^{m} \mathbb{P}\left(f_{j} \mid e_{i}\right)=\frac{1}{m} \mathbb{P}\left(\sum_{j=1}^{m} f_{j} \mid e_{i}\right)=\frac{1}{m}
$$

for $i=1, \ldots, l$. Therefore 


$$
1=\mu\left(\sum_{j=1}^{m} f_{j}\right) \geq \mu\left(\sum_{i=1}^{l} e_{i}\right)=\sum_{i=1}^{l} \mu\left(e_{i}\right)=\frac{l}{m}
$$

and thus $l \leq m$.

(iii) Let $0 \neq p \in L$. If there is no $q_{1} \in L$ with $0 \neq q_{1} \leq p$ and $q_{1} \neq p$, the proposition $p$ itself is minimal and we are done. In the other case, consider $p \wedge q_{1}{ }^{\prime} \neq 0$. Either $p \wedge q_{1}{ }^{\prime}$ is minimal and we are done again or there is a proposition $q_{2} \in L$ with $0 \neq q_{2} \leq p \wedge q_{1}{ }^{\prime}$ and $q_{2} \neq p \wedge q_{1}{ }^{\prime}$. This procedure is continued, but must stop after a finite number of steps by (i), since the $q_{1}, q_{2}, \ldots$ are pairwise orthogonal.

(iv) Let $0 \neq p \in L$. By (iii), there is a minimal proposition $e_{1}$ with $e_{1} \leq p$. If $e_{1}=p$, we are done. If not, consider $p \wedge e_{1}{ }^{\prime} \neq 0$ and again apply (ii) to get a minimal proposition $e_{2}$ with $e_{2} \leq p \wedge e_{1}{ }^{\prime}$. If $e_{2}=p \wedge e_{1}{ }^{\prime}$, we have $p=e_{1}+e_{2}$. If not, continue this procedure. By (i), it stops again after a finite number of steps, since the $e_{1}, e_{2}, \ldots$ are pairwise orthogonal.

(v) By (iv), I is the sum of a finite number of pairwise orthogonal minimal propositions $e_{1}, \ldots, e_{n}$. Define a state $\tau$ by

$$
\tau(q):=\frac{1}{n} \sum_{k=1}^{n} \mathbb{P}\left(q \mid e_{k}\right) \text { for } q \in L .
$$

For any minimal proposition $f$ in $L$ we then have by postulate $(\mathrm{C})$

$$
\tau(f)=\frac{1}{n} \sum_{k=1}^{n} \mathbb{P}\left(f \mid e_{k}\right)=\frac{1}{n} \sum_{k=1}^{n} \mathbb{P}\left(e_{k} \mid f\right)=\frac{1}{n} \mathbb{P}\left(\sum_{k=1}^{n} e_{k} \mid f\right)=\frac{1}{n} \mathbb{P}(\mathbb{I} \mid f)=\frac{1}{n} .
$$

Now assume that $\rho$ is a further state allocating identical values to the minimal propositions. Then $1=\rho(\mathbb{I})=n \rho\left(e_{1}\right)$ and thus $\rho(f)=1 / n$ for all minimal propositions $f$. Since all propositions are sums of minimal propositions by (iv), $\rho$ must coincide with $\tau$.

(vi) Let $p \in L$. By (iv), $p=\sum_{k=1}^{m} e_{k}$ and $p^{\prime}=\sum_{k=m+1}^{n} e_{k}$ with $n$ pairwise orthogonal minimal propositions $e_{1}, \ldots, e_{n}$. Then $\mathbb{I}=p+p^{\prime}=\sum_{k=1}^{n} e_{k}$. By (v), $\tau(q)=\frac{1}{n} \sum_{k=1}^{n} \mathbb{P}\left(q \mid e_{k}\right)$ for $q \in L$. It shall now be shown that the following two identities hold for $q \in L$ :

$$
\tau(q \mid p)=\frac{1}{m} \sum_{k=1}^{m} \mathbb{P}\left(q \mid e_{k}\right)
$$

and

$$
\tau\left(q \mid p^{\prime}\right)=\frac{1}{n-m} \sum_{k=1}^{m} \mathbb{P}\left(q \mid e_{k}\right) .
$$

If $q$ is a minimal proposition with $q \leq p$, the right-hand side of (4.1) yields, by postulate (C),

$$
\begin{aligned}
\frac{1}{m} \sum_{k=1}^{m} \mathbb{P}\left(q \mid e_{k}\right) & =\frac{1}{m} \sum_{k=1}^{m} \mathbb{P}\left(e_{k} \mid q\right)=\frac{1}{m} \mathbb{P}\left(\sum_{k=1}^{m} e_{k} \mid q\right) \\
& =\frac{1}{m} \mathbb{P}(p \mid q)=\frac{1}{m}=\frac{\tau(q)}{\tau(p)}=\tau(q \mid p) .
\end{aligned}
$$


Note that $\tau(q)=1 / n$ and $\tau(p)=m / n$. Identity (4.1) then follows for all propositions $q \leq p$, since each such $q$ is a sum of minimal propositions, and the uniqueness of the conditional probability implies that it holds for all propositions $q$ in $L$. Identity (4.2) follows in the same way. With (4.1) and (4.2), we then have, for any proposition $q$ in $L$,

$$
\begin{aligned}
\tau(q \mid p) \tau(p)+\tau\left(q \mid p^{\prime}\right) \tau\left(p^{\prime}\right) & =\tau(q \mid p) \frac{m}{n}+\tau\left(q \mid p^{\prime}\right)\left(1-\frac{m}{n}\right) \\
& =\frac{1}{n} \sum_{k=1}^{n} \mathbb{P}\left(q \mid e_{k}\right)=\tau(q) .
\end{aligned}
$$

\section{Results}

In this section, some earlier results of [44] will be needed; they are restated in the following two lemmas for the specific finite-dimensional case considered here.

Proposition 5.1. Let $L$ be a quantum logic satisfying the three postulates (A), (B) and (D). Then

(i) $V$ is a base-norm space.

(ii) $L$ can be embedded in the dual of $V$, which is an order-unit space denoted by $A$, in such a way that $A$ is the linear hull of $L$, each element of $L$ is positive in $A$ and the order relations on $L$ and $A$ coincide.

(iii) There is an idempotent positive linear map $U_{p}: A \rightarrow A$ for each $p \in L \subseteq A$ such that the conditional probabilities have the shape

$$
\mu(q \mid p) \mu(q)=\mu\left(U_{p} q\right)
$$

for any state $\mu$ on $L$ and any proposition $q$ in $L$ (note that $\mu \in V$ is identified with its canonical embedding in the second dual $V^{* *}=A^{*}$ here and in the following; because of the finite dimension of $V$, we actually have $V=V^{* *}=A^{*}$ ). 1

(iv) There is a bilinear operation $A \times A \rightarrow A,(a, b) \mapsto a \square b$ with

$$
p \square q=\frac{1}{2}\left(q+U_{p} q-U_{p^{\prime}} q\right),
$$

for $p, q \in L$.

(v) $p^{2}:=p \square p=p$ for any $p \in L$ and $p \square q=0$ for any two orthogonal propositions $p, q \in L$.

\footnotetext{
${ }^{1}$ These $U_{p}$ projections bear some similarities to the so-called compressions introduced and used by Alfsen and Shultz [1], but lack an important feature to become compressions: $U_{p} q=0$ does not imply $U_{p^{\prime}} q=q$ for $p, q \in L$.
} 
The first three items of this proposition follow from theorem 3.2 in [44]; (iv) and (v) are shown in section 10 in [44. Note that the so-called $\epsilon$-Hahn-Jordan decomposition property, needed there, immediately follows from postulate (B). Moreover, note that the orthomodular partially ordered set $L$ satisfies the slightly more general definition of a quantum logic used in [44. Furthermore, because of the finite dimension of $V$, its dual $A$ is finite-dimensional as well; this is why the different topologies used in 44 need not be considered here, why all linear subspaces become closed and why all linear maps are continuous.

Proposition 5.2. Let $L$ be a quantum logic satisfying the three postulates (A), (B) and (D). If the bilinear operation $\square$ of proposition 5.1 is power-associative and the squares $a^{2}:=a \square a, a \in A$, are positive elements in the order-unit space $A$, then the bilinear operation $\square$ is commutative and $A$ is a formally real Jordan algebra.

This immediately follows from theorem 11.1 in [44]; in its proof, it is mentioned that $A$ becomes a so-called JBW/JB-algebra and note that, in the finitedimensional case considered here, the JBW/JB-algebras are identical to the formally real Jordan algebras.

Theorem 5.3. A quantum logic L which satisfies the four postulates (A), (B), (C) and (D) is identical to the quantum logic formed by the idempotent elements of a finite-dimensional formally real Jordan algebra.

Proof. Let $L$ be a quantum logic which satisfies the four postulates (A), (B), (C) and (D). Lemma 4.1 (iv) implies that $\mathbb{I}$ is the sum of a finite number of orthogonal minimal propositions; the propositions vary, but lemma 4.1 (ii) implies that their number is fixed and is a typical characteristic of the quantum logic $L$. In the following, let this be $n$. By lemma $4.1(\mathrm{v})$, it follows that $\tau(e)=1 / n$ for the trace state $\tau$ and all minimal propositions $e \in L$.

For any two minimal propositions $e$ and $f$ in $L$, we get, using proposition 5.1 (iii) and (iv) for the first equality, lemma 4.1 (vi) for the second one, Definition 2.2 for the third one and finally postulate $(\mathrm{C})$ for the last one,

$$
\begin{aligned}
\tau(f \square e) & =\frac{1}{2}\left(\tau(f)+\tau(f \mid e) \tau(e)-\tau\left(f \mid e^{\prime}\right) \tau\left(e^{\prime}\right)\right) \\
& =\tau(f \mid e) \tau(e)=\mathbb{P}(f \mid e) \tau(e)=\mathbb{P}(f \mid e) / n=\mathbb{P}(e \mid f) / n
\end{aligned}
$$

and thus $\tau(f \square e)=\tau(e \square f)$. Since the minimal propositions generate $A$, we get

$$
\tau(a \square b)=\tau(b \square a) \text { for all } a, b \in A
$$

and

$$
\tau(e \square p)=\tau(p \square e)=\mathbb{P}(p \mid e) / n \text { for any } p, e \in L, \text { with } e \text { being minimal. }
$$

For any $x \in A$ define $\tau_{x} \in V$ by $\tau_{x}(p):=\tau(x \square p), p \in L$. Owing to postulate (B), each $\mu \in V$ has this shape, which can be seen in the following way: there 
are orthogonal minimal propositions $e_{1}, \ldots, e_{m}$, real numbers $r_{1}, \ldots, r_{m}$ and some non-negative integer $m$ such that

$$
\mu(p)=\sum_{k=1}^{m} r_{k} \mathbb{P}\left(p \mid e_{k}\right)=n \sum_{k=1}^{n} r_{k} \tau\left(e_{k} \square p\right)
$$

for $p \in L$; this means $\mu=\tau_{a}$ with

$$
a=n \sum_{k=1}^{m} r_{k} e_{k} \in A
$$

Now consider $\tau_{b} \in V$ with any $b \in A$. Then there is some $a \in A$ with the above shape and $\tau_{b}=\tau_{a}$. For all $x \in A$, it follows that

$$
\begin{aligned}
\tau_{x}(b) & =\tau(x \square b)=\tau(b \square x)=\tau_{b}(x) \\
& =\tau_{a}(x)=\tau(a \square x)=\tau(x \square a)=\tau_{x}(a) .
\end{aligned}
$$

Since each $\mu \in V$ has the shape $\mu=\tau_{x}$ with some $x \in A$, this means $\mu(b)=\mu(a)$ for all $\mu \in V$ and finally $b=a$. Therefore, each $b \in A$ is the linear combination of orthogonal propositions from $L$ (i.e., $b$ has a spectral decomposition).

Now suppose $b=\sum_{k=1}^{m} s_{k} p_{k} \in A$ with orthogonal propositions $p_{1}, \ldots, p_{m} \neq 0$ and real numbers $s_{1}, \ldots, s_{m}$. Then by proposition $5.1(\mathrm{v})$ and (ii)

$$
b^{2}:=b \square b=\sum_{k=1}^{m} s_{k}^{2} p_{k} \geq 0
$$

and, with $b^{l+1}:=b \square b^{l}$ for positive integers $l$,

$$
b^{l}=\sum_{k=1}^{m} s_{k}^{l} p_{k} .
$$

This means that the square of each element in $A$ is a positive element in the order-unit space $A$ and that the bilinear operation $\square$ is power-associative. By proposition 5.2, it is commutative and $A$ becomes a formally real Jordan algebra.

Now assume $b=b^{2}$. Then

$$
\sum_{k=1}^{m} s_{k} p_{k}=\sum_{k=1}^{m} s_{k}^{2} p_{k} .
$$

The linear independence of $p_{1}, \ldots, p_{m}$ implies $s_{k}=s_{k}^{2}$ and hence $s_{k}=0$ or $s_{k}=1$ for $k=1, \ldots, m$. Therefore, $b$ is the sum of orthogonal propositions in $L$ and thus itself an element of $L$. This means $L=\left\{b \in A: b^{2}=b\right\}$.

Combining theorem 5.3 with propositions 3.1 and 3.3 immediately yields the following corollary.

Corollary 5.4. A quantum logic L satisfies the four postulates (A), (B), (C) and (D) if and only if it is identical to the system of the idempotent elements in a finite-dimensional formally real Jordan algebra whose irreducible subalgebras do not not include spin factors. 


\section{Conclusion}

\section{(a) Reconstruction of quantum mechanics}

Except for the so-called exceptional Jordan algebras, each formally real Jordan algebra with finite dimension has a representation as a Jordan subalgebra of the self-adjoint operators on a unitary space (or, equivalently, self-adjoint quadratic matrices with complex entries). The above results thus come close to a derivation of the finite-dimensional case of quantum theory's mathematical apparatus from the four postulates (A), (B), (C) and (D). Unfortunately, this is not a complete reconstruction of quantum mechanics, since there remain two problems. The first one is that the four postulates (A), (B), (C) and (D) rule out the spin factors and thus a certain part of standard quantum mechanics: the two-dimensional Hilbert space and therefore also the qubit. The second problem is that the four postulates (A), (B), (C) and (D) allow for the exceptional Jordan algebras, which are not part of standard quantum mechanics.

Concerning the first problem, note that only the isolated single qubit is ruled out; the quantum-mechanical model of an $n$-qubit system is the algebra of $2^{n} \times 2^{n}$-matrices with complex entries and its self-adjoint part (with the special Jordan product $x \circ y:=(x y+y x) / 2)$ satisfies the postulates $(\mathrm{A}),(\mathrm{B}),(\mathrm{C})$ and (D) for $n \neq 1$.

Some approaches to overcome the second problem and to find reasons for the necessity of the complex numbers in quantum mechanics are:

(1) dynamical correspondence [1, 47]

(2) energy observable assignment 7 ]

(3) local tomography [5, 8, 9, 13, 16, 27, 35]

(4) 3-ball property 1

(5) orientations [1, 2].

The first two approaches are closely related. Both concern the mathematical model of the dynamical evolution of a single system and are motivated by the quantum-mechanical feature that the dynamical group is generated by the Hamilton operator.

Local tomography means that one can estimate the state of a composite system, composed of two or more subsystems, by making measurements on its subsystems (taking into account their correlations). Local tomography could be phrased as an additional postulate directly in the quantum logical setting of the present paper, while this would be hard to do for the other approaches. Though not explicitly stated in this way, the results in [35] appear to imply that local tomography distinguishes the complex case among the irreducible (simple) finite-dimensional formally real Jordan algebras [5], but it seems to be unknown whether this holds generally when the reducible (non-simple) algebras are also considered.

The last two approaches involve mathematically interesting concepts, but a physical motivation is hard to find for them. 


\section{(b) Potential generalizations}

A different interesting problem is the question of what opportunities there are for a physically meaningful generalization of quantum theory's mathematical apparatus. The conditional probabilities represent an extension of projective quantum measurement to the quantum logical setting and this may be a good reason to maintain postulate $(\mathrm{A})$.

Moreover, it has been shown in 44 that the non-existence of third-order interference is responsible for the existence of the operation $\square$ used in the proof of the above theorem, and in [46] a close link between this operation and the existence of continuous symmetries (Lie groups) has been elaborated. A potential physically meaningful generalization of quantum mechanics should include continuous symmetries, and this may be an important reason to adhere to postulate (D) as well.

Therefore, only postulates (B) and (C) leave room for a potential generalization of quantum theory's mathematical apparatus. The orthogonality of the minimal propositions $e_{1}, \ldots, e_{m}$ in postulate $(\mathrm{B})$ is a very strong requirement and becomes one candidate to dispense with. The symmetry of the transition probabilities is another candidate, although it is often taken for granted. Some of the rare cases where asymmetric transition probabilities were considered in the past are [23, 36, 37.

The continuous symmetry groups of the finite-dimensional simple formally real Jordan algebras cover all the non-exceptional compact simple Lie groups. Only five exceptional compact simple Lie groups remain; these are $G_{2}, F_{4}, E_{6}$, $E_{7}, E_{8}$. However, $F_{4}$ is also covered, since it is the Lie group of the exceptional Jordan algebra formed by the Hermitian $3 \times 3$-matrices over the octonions, and $G_{2}$ is the Lie group of the octonions themselves [4. Therefore, only $E_{6}, E_{7}$ and $E_{8}$ are not related to the symmetries of the formally real Jordan algebras and their associated quantum logics. Dropping postulate (B) or (C) or both may thus provide opportunities to search for quantum logics with an $E_{6}, E_{7}$ or $E_{8}$ symmetry.

\section{(c) Soundness of plausibility considerations}

Deriving the mathematical apparatus of quantum mechanics from a small number of mathematical features of this theory helps, on the one hand, to better understand why this apparatus is required and, on the other hand, to study potential generalizations which might provide opportunities for future progress in theoretical physics. These features may be more or less physically motivated and those with less motivation become the first candidates to dispense with in a potential generalization. However one must be careful. The implications of such a feature may not be obvious immediately and should be studied in the mathematical framework first. Moreover, physical plausibility depends on a sci-

entist's personal background and belief. Could quantum theory ever have been detected on the basis of physical plausibility in the nineteenth century? 
At that time one would have insisted on commutative algebras for the observables and Boolean lattices for the propositions. Only later experimental evidence enforced the use of non-commutative algebras, resulting in the nonclassical quantum logics.

The conditional probabilities considered in this paper are the only possible extension of the classical ones to quantum logics and, for the standard Hilbert space quantum logic, it turns out that probability conditionalization is identical to the state transition of projective quantum measurement. Its behavior is far away from any plausibility considerations from a classical point of view.

Hilbert space quantum theory includes second-order interference, but excludes third- and higher-order interference. At first glance, there are no plausible reasons why third- and higher-order interference should be ruled out, while second-order interference is allowed. However, studying the implications in a generic mathematical framework reveals a link to the existence of continuous symmetries, which are quite important in physical theories.

Physically convincing reasons for the symmetry of the transition probabilities (C) and for postulate (B) seem to be harder to find. Both require minimal propositions which do not exist in some infinite-dimensional operator algebras used in quantum statistical mechanics and in quantum field theory. The general validness of these two postulates in their current form is thus ruled out by physical theories that already exist.

\section{References}

[1] E. M. Alfsen and F. W. Shultz. Geometry of state spaces of operator algebras. Boston, MA: Birkhäuser, 2012.

[2] E. M. Alfsen and F. W. Shultz. State spaces of operator algebras: basic theory, orientations, and $C^{*}$-products. Boston, MA: Birkhäuser, 2012.

[3] H. Araki. On a characterization of the state space of quantum mechanics. Communications in Mathematical Physics, 75(1):1-24, 1980.

[4] J. Baez. The octonions. Bulletin of the American Mathematical Society, 39(2):145-205, 2002.

[5] H. Barnum and J. Hilgert. Strongly symmetric spectral convex bodies are Jordan algebra state spaces. arXiv eprint math-ph:1904.03753, 2019.

[6] H. Barnum, C. M. Lee, C. M. Scandolo, and J. H. Selby. Ruling out higherorder interference from purity principles. Entropy, 19(6), 2017.

[7] H. Barnum, M. P. Müller, and C. Ududec. Higher-order interference and single-system postulates characterizing quantum theory. New Journal of Physics, 16(12):123029, 2014.

[8] H. Barnum and A. Wilce. Local tomography and the Jordan structure of quantum theory. Foundations of Physics, 44(2):192212, Feb 2014. 
[9] J. Barrett. Information processing in generalized probabilistic theories. Physical Review A, 75(3):032304, 2007.

[10] G. Birkhoff and J. von Neumann. The logic of quantum mechanics. Annals of Mathematics, 37:823-843, 1936.

[11] J. Bub. Von Neumann's projection postulate as a probability conditionalization rule in quantum mechanics. Journal of Philosophical Logic, 6(1):381-390, 1977.

[12] L. Bunce and J. M. Wright. Quantum measures and states on Jordan algebras. Communications in Mathematical Physics, 98(2):187-202, 1985.

[13] G. Chiribella, G. M. DAriano, and P. Perinotti. Informational derivation of quantum theory. Physical Review A, 84(1):012311, 2011.

[14] B. Dakić and Č. Brukner. Quantum theory and beyond: Is entanglement special? In H. Halvorson, editor, Deep Beauty: Understanding the Quantum World through Mathematical Innovation, pages 365-392. Cambridge, UK: Cambridge University Press, 2011.

[15] B. Dakić, T. Paterek, and Č. Brukner. Density cubes and higher-order interference theories. New Journal of Physics, 16(2):023028, 2014.

[16] G. de la Torre, L. Masanes, A. J. Short, and M. P. Müller. Deriving quantum theory from its local structure and reversibility. Physical Review Letters, 109(9):090403, 2012.

[17] D. I. Fivel. Derivation of the rules of quantum mechanics from informationtheoretic axioms. Foundations of Physics, 42(2):291-318, Oct 2011.

[18] M. Friedman and H. Putnam. Quantum logic, conditional probability, and interference. Dialectica, 32(3-4):305-315, 1978.

[19] A. M. Gleason. Measures on the closed subspaces of a Hilbert space. Journal of Mathematics and Mechanics, 6:885-893, 1957.

[20] P. Goyal. From information geometry to quantum theory. New Journal of Physics, 12(2):023012, 2010.

[21] J. Gunson. On the algebraic structure of quantum mechanics. Communications in Mathematical Physics, 6(4):262-285, 1967.

[22] W. Guz. Conditional probability in quantum axiomatics. Annales de l'IHP Physique Théorique, 33(1):63-119, 1980.

[23] W. Guz. A non-symmetric transition probability in quantum mechanics. Reports on Mathematical Physics, 17(3):385-400, 1980.

[24] W. Guz. Conditional probability and the axiomatic structure of quantum mechanics. Fortschritte der Physik, 29(8):345-379, 1981. 
[25] H. Hanche-Olsen and E. Størmer. Jordan operator algebras. Boston, MA: Pitman Advanced Publishing Program, vol. 21, 1984.

[26] L. Hardy. Quantum theory from five reasonable axioms. arXiv eprint quant-ph/0101012, 2001.

[27] L. Hardy. Foliable operational structures for general probabilistic theories. In H. Halvorson, editor, Deep Beauty: Understanding the Quantum World through Mathematical Innovation, pages 409-442. Cambridge, UK: Cambridge University Press, 2011.

[28] J. Hickmann, E. Fonseca, and A. Jesus-Silva. Born's rule and the interference of photons with orbital angular momentum by a triangular slit. EPL (Europhysics Letters), 96(6):64006, 2011.

[29] P. A. Höhn and C. S. P. Wever. Quantum theory from questions. Physical Review A, 95:012102, 2017.

[30] H. A. Keller. Ein nicht-klassischer Hilbertscher Raum. Mathematische Zeitschrift, 172(1):41-49, 1980.

[31] C. M. Lee and J. H. Selby. Deriving Grover's lower bound from simple physical principles. New Journal of Physics, 18(9):093047, 2016.

[32] M. S. Leifer and R. W. Spekkens. Towards a formulation of quantum theory as a causally neutral theory of Bayesian inference. Physical Review A, 88(5):052130, 2013.

[33] G. Ludwig. Foundations of quantum mechanics, vols. I and II. Texts and Monographs in Physics. Berlin, Germany: Springer, 1983 and 1986.

[34] G. W. Mackey. Lecture notes on the mathematical foundations of quantum mechanics: Math 265. Boston, MA: Harvard University, 1960.

[35] L. Masanes and M. P. Müller. A derivation of quantum theory from physical requirements. New Journal of Physics, 13(6):063001, 2011.

[36] B. Mielnik. Theory of filters. Communications in Mathematical Physics, 15(1):1-46, 1969.

[37] B. Mielnik. Generalized quantum mechanics. Communications in Mathematical Physics, 37(3):221-256, 1974.

[38] O. Nánásiová. Conditional probability on a quantum logic. International journal of theoretical physics, 25(11):1155-1162, 1986.

[39] O. Nánásiová. Representation of conditional probability on a quantum logic. Soft Computing, 4(1):36-40, 2000.

[40] O. Nánásiová and M. Kalina. Calculus for non-compatible observables, construction through conditional states. International Journal of Theoretical Physics, 54(2):506-518, 2015. 
[41] O. Nánásiová and L. Valášková. Maps on a quantum logic. Soft Computing, 14(10):1047-1052, 2010.

[42] G. Niestegge. Non-Boolean probabilities and quantum measurement. Journal of Physics A: Mathematical and General, 34(30):6031, 2001.

[43] G. Niestegge. An approach to quantum mechanics via conditional probabilities. Foundations of Physics, 38(3):241-256, 2008.

[44] G. Niestegge. Conditional probability, three-slit experiments, and the Jordan algebra structure of quantum mechanics. Advances in Mathematical Physics, 2012, 2012.

[45] G. Niestegge. Three-slit experiments and quantum nonlocality. Foundations of Physics, 43(6):805-812, 2013.

[46] G. Niestegge. A generalized quantum theory. Foundations of Physics, 44(11):1216-1229, 2014.

[47] G. Niestegge. Dynamical correspondence in a generalized quantum theory. Foundations of Physics, 45(5):525-534, 2015.

[48] D. K. Park, O. Moussa, and R. Laflamme. Three path interference using nuclear magnetic resonance: A test of the consistency of Born's rule. New Journal of Physics, 14(11):113025, 2012.

[49] C. Piron. Axiomatique quantique. Helvetica Physica Acta, 37(4-5):439-468, 1964.

[50] J. C. Pool. Baer*-semigroups and the logic of quantum mechanics. Communications in Mathematical Physics, 9(2):118-141, 1968.

[51] S. Pulmannová. Quantum conditional probability spaces. In L. Accardi and W. von Waldenfels, editors, Quantum Probability and Applications V, pages 320-325. Lecture Notes in Mathematics, vol. 1442. Berlin, Germany: Springer, 1990.

[52] M. Rédei. Quantum Logic in Algebraic Approach. Dordrecht, The Netherlands: Kluwer Academic Publishers, 1998.

[53] I. E. Segal. Postulates for general quantum mechanics. Annals of Mathematics, pages 930-948, 1947.

[54] J. H. Selby, C. M. Scandolo, and B. Coecke. Reconstructing quantum theory from diagrammatic postulates. arXiv eprint quant-ph/1802.00367, 2018.

[55] S. Sherman. On Segal's postulates for general quantum mechanics. Annals of Mathematics, pages 593-601, 1956. 
[56] U. Sinha, C. Couteau, T. Jennewein, R. Laflamme, and G. Weihs. Ruling out multi-order interference in quantum mechanics. Science, 329(5990):418-421, 2010.

[57] M. P. Soler. Characterization of Hilbert spaces by orthomodular spaces. Communications in Algebra, 23(1):219-243, 1995.

[58] I. Söllner, B. Gschösser, P. Mai, B. Pressl, Z. Vörös, and G. Weihs. Testing Born's rule in quantum mechanics for three mutually exclusive events. Foundations of Physics, 42(6):742-751, 2012.

[59] R. D. Sorkin. Quantum mechanics as quantum measure theory. Modern Physics Letters A, 9(33):3119-3127, 1994.

[60] S. Tull. A categorical reconstruction of quantum theory. arXiv eprint quant-ph/1804.02265, 2018.

[61] J. van de Wetering. An effect-theoretic reconstruction of quantum theory. arXiv eprint quant-ph/1801.05798, 2018.

[62] V. S. Varadarajan. Geometry of Quantum Theory, vols. 1 and 2. New York, NY: Van Nostrand Reinhold, 1968 and 1970.

[63] J. von Neumann, P. Jordan, and E. Wigner. On an algebraic generalization of the quantum mechanical formalism. Annals of Mathematics, 35:29-64, 1934.

[64] A. Wilce. Four and a half axioms for finite-dimensional quantum probability. In Y. Ben-Menahem and M. Hemmo, editors, Probability in Physics, pages 281-298. The Frontiers Collection. Berlin, Germany: Springer, 2012. 